\title{
Dexmedetomidine on autophagy of hippocampal neurons in aged rats under sevoflurane anesthesia
}

\author{
CHUNFANG YI ${ }^{1}$, ZHIYUN FU $^{2}$ and XIAOFENG LUO ${ }^{3}$ \\ Departments of ${ }^{1}$ Anesthesiology and ${ }^{2}$ Respiratory Medicine, The First Affiliated Hospital of \\ Nanchang University, Nanchang, Jiangxi 330006; ${ }^{3}$ Department of Anesthesiology, \\ People's Hospital of Yifeng, Yifeng, Jiangxi 336300, P.R. China
}

Received September 1, 2017; Accepted May 15, 2018

DOI: $10.3892 / \mathrm{etm} .2018 .6219$

\begin{abstract}
Dexmedetomidine is a highly-selective $\alpha$ adrenergic receptor agonist, widely used as an anesthesia adjuvant drug in clinic. Effects of dexmedetomidine on autophagy of hippocampal neurons and cognitive dysfunction in aged rats under sevoflurane anesthesia were investigated. Sixty healthy Sprague-Dawley (SD) rats aged 4-6 months of either sex, weighing 150-200 $\mathrm{g}$ were selected and randomly divided into control group $(n=10)$, sevoflurane group $(n=25)$ and dexmedetomidine + sevoflurane group (compound group, $\mathrm{n}=25$ ). The control group was inhaled with $60 \% \mathrm{O}_{2}$ for $6 \mathrm{~h}$, the sevoflurane group was inhaled with 3.4-3.6\% sevoflurane for $6 \mathrm{~h}$, and the compound group was treated with intraperitoneal injection of $4 \mu \mathrm{g} / \mathrm{kg}$ dexmedetomidine $1 \mathrm{~h}$ before the inhalation of sevoflurane. Five rats were taken from each group $1 \mathrm{~h}$ before sevoflurane anesthesia (T1), immediately after anesthesia (T2), $12 \mathrm{~h}$ after anesthesia (T3) and $24 \mathrm{~h}$ after anesthesia (T4), respectively. The expression levels of microtubule-associated protein 1 light-chain 3-I (LC3-I), LC3-II and Beclin-1 were detected via western blotting, and the LC3-II/LC3-I value was calculated. Moreover, Morris water maze test was performed for the five rats in each group 5 weeks after anesthesia to detect the cognitive function; the escape latency, times across platform and swimming time in target quadrant were recorded. The levels of LC3-I, LC3-II and Beclin-1 in hippocampal neurons and LC3-II/LC3-I value in sevoflurane group at T2 were significantly higher than those at $\mathrm{T} 1$, and they reached the peak at $\mathrm{T} 3$, but were decreased at $\mathrm{T} 4(\mathrm{P}<0.05)$; the levels of proteins and LC3-II/LC3-I value in compound group at $\mathrm{T} 2$ were significantly higher than those at $\mathrm{T} 1$ and reached the peak, and remained unchanged at T3-T4; besides, the level of proteins and LC3-II/LC3-I values in compound group at T2-T4
\end{abstract}

Correspondence to: Dr Chunfang Yi, Department of Anesthesiology, The First Affiliated Hospital of Nanchang University, 17 Yongwaizheng Street, Nanchang, Jiangxi 330006, P.R. China E-mail: yichunfang17@163.com

Key words: dexmedetomidine, sevoflurane, cognitive dysfunction, hippocampal neurons, autophagy were obviously lower than those in sevoflurane group $(\mathrm{P}<0.05)$. In sevoflurane group, the escape latency was prolonged, the times across platform were reduced and the swimming time in target quadrant was shortened compared with those in control group $(\mathrm{P}<0.05)$; these parameters were significantly improved in compound group compared with those in sevoflurane group and control group $(\mathrm{P}<0.05)$. In conclusion, dexmedetomidine can improve the cognitive dysfunction in aged rats under sevoflurane anesthesia, which is related to the decreased autophagy of hippocampal neurons.

\section{Introduction}

Sevoflurane is a kind of commonly-used inhalational general anesthetics. A study has pointed out (1) that sevoflurane can lead to the decline in cognitive function in the recovery period, which may be related to the surgical trauma, anesthesia depth, body inflammation, stress and immune dysfunction. The elderly patients are often complicated with low metabolic function, poor operation and anesthesia tolerance, and many perioperative complications, so the postoperative cognitive dysfunction (POCD) occurs more easily after operation (2). Hippocampus is an important brain area involved in learning and memory ability, and the pathological changes, such as $\beta$-amyloid protein deposit and Tau protein phosphorylation, caused by hippocampal neurons are the important mechanisms of Alzheimer's disease (3); therefore, it is assumed that POCD caused by sevoflurane anesthesia may also be related to abnormalities in hippocampal neuronal structure and function. Cell autophagy is an important way for eukaryotes to degrade the misfolded proteins and damaged organelles in the body via lysosomes, and maintain the homeostasis, which is also known as type II programmed death (4). The decreased autophagy is associated with tumorigenesis (4), while the enhanced autophagy is related to the occurrence of neurodegenerative disease (5). Autophagosomes and specific proteins light-chain 3-I (LC3-I), LC3-II and Beclin-1 can reflect the autophagic activity of the body. Dexmedetomidine is a highly-selective $\alpha$ adrenergic receptor agonist, widely used as an anesthesia adjuvant drug in clinic, which is characterized by rapid onset of analgesia and sedation, small side effects, little inhibition on respiration and circulation and high safety $(6,7)$. This study aimed to analyze the effects of dexmedetomidine on 
autophagy of hippocampal neurons and cognitive dysfunction in aged rats under sevoflurane anesthesia.

\section{Materials and methods}

Animals. Sixty healthy Sprague-Dawley (SD) rats aged 4-6 months of either sex, weighing 150-200 g were purchased from Laboratory Animal Center of Jilin University (certificate no.: SCXK (Ji) 2008-0004). They ate and drank freely at room temperature of $22 \pm 2^{\circ} \mathrm{C}$ and relative humidity of $55 \pm 2 \%$ to adapt to the environment for 1 week, followed by subsequent experiments. The study was approved by the Animal Ethics Committee of Nanchang University (Nanchang, China).

Research methods. The rats were randomly divided into control group $(n=10)$, sevoflurane group $(n=25)$ and dexmedetomidine + sevoflurane group (compound group, $\mathrm{n}=25$ ). The control group was inhaled with $60 \% \mathrm{O}_{2}$ for $6 \mathrm{~h}$, the sevoflurane group was inhaled with 3.4-3.6\% sevoflurane for $6 \mathrm{~h}$, and the compound group was treated with intraperitoneal injection of $4 \mu \mathrm{g} / \mathrm{kg}$ dexmedetomidine $1 \mathrm{~h}$ before the inhalation of sevoflurane. Five rats were taken from each group before sevoflurane anesthesia (T1), immediately after anesthesia (T2), $12 \mathrm{~h}$ after anesthesia (T3) and $24 \mathrm{~h}$ after anesthesia (T4). The expression levels of microtubule-associated protein 1 LC3-I, LC3-II and Beclin-1 were detected via western blotting, and the LC3-II/ LC3-I value was calculated. Moreover, Morris water maze test was performed for the five rats in each group 5 weeks after anesthesia to detect the cognitive function; the escape latency, times across platform and swimming time in target quadrant were recorded.

Materials. The rats were placed in a plastic airtight transparent container, then the container was placed in the ZD-600 thermostatic water bath to maintain the temperature of $37^{\circ} \mathrm{C}$, and the bottom of the container was covered with calcium lime to absorb the $\mathrm{CO}_{2}$ generated; two side walls of the container were the air inlet and exhaust port; the air inlet was connected to the Vapor 2000 anesthetic gas volatilization pot (Dreager, Lübeck, Germany) for oxygen or sevoflurane delivery (Jiangsu Hengrui Medicine Co., Ltd., Jiangsu, China), while the exhaust port was connected to the Vamos multifunctional anesthetic gas monitor (Dreager) for monitoring the sevoflurane and $\mathrm{CO}_{2}$ concentrations; the abdomen of rats was connected to the probe of oxygen saturation monitor (Philips Medical Systems B.V., Eindhoven, The Netherlands).

Western blotting. After anesthesia using chloral hydrate via intraperitoneal injection at a dose of $300-350 \mathrm{mg} / \mathrm{kg}$, the rats were sacrificed and the brain was removed, the hippocampus tissues were separated and placed in liquid nitrogen at $-80^{\circ} \mathrm{C}$. After hippocampus tissue homogenate, the centrifugation was performed at $4^{\circ} \mathrm{C}$ at $10,500 \mathrm{xg}$ for $15 \mathrm{~min}$; the supernatant was taken, and the protein concentration was detected using the bicinchoninic acid (BCA) protein quantification kit (Jiangsu Nanjing Beyotime Technology Co., Ltd., Jiangsu, China). The loading buffer was added, followed by $12 \%$ sodium dodecyl sulfate polyacrylamide gel electrophoresis (SDS-PAGE); the protein was transferred onto the polyvinylidene fluoride (PVDF) membrane. After sealing with blocking buffer at $37^{\circ} \mathrm{C}$ for $1 \mathrm{~h}$, rabbit polyclonal LC3-I (dilution: 1:2,000; cat. no. ab48394), rabbit polyclonal LC3-II (dilution: 1:2,000; cat. no. ab192890), rabbit polyclonal Beclin-1 (dilution: 1:2,000; cat. no. ab207612) and rabbit polyclonal GAPDH (dilution: 1:500, cat. no. ab37168), purchased from Abcam (Cambridge, MA, USA), were added at $4^{\circ} \mathrm{C}$ overnight. After washing, the goat anti-mouse polyclonal secondary goat anti-mouse (HRP) IgG antibody (dilution: 1:2,000; cat. no. ab6789; Abcam, Cambridge, MA, USA) was incubated at $37^{\circ} \mathrm{C}$ for $4 \mathrm{~h}$, followed by washing via phosphate buffered saline (PBS) and ECL development. The results were scanned and saved, and the semi-quantitative analysis was performed using the Lab Works4.5 gel imaging software (Invitrogen; Thermo Fisher Scientific, Inc.).

Morris water maze test. Morris water maze test included the place navigation and space exploration. In place navigation test, the platform was placed in the third quadrant, and the rats stood on the platform for $30 \mathrm{sec}$; the water was poured into the pool wall from different quadrants, and the time of finding and climbing on platform within $60 \mathrm{sec}$ (escape latency) was recorded. After finding the platform, the rats stood for $30 \mathrm{sec}$ and rested. If the rats failed to find the platform within $60 \mathrm{sec}$, they were led onto the platform to stay for $15 \mathrm{sec}$, and the escape latency was recorded as $60 \mathrm{sec}$. The test was repeated 4 times per day for 5 days, and the average was taken. In space exploratory test, the platform was removed, and the rat was put into the water optionally from the same entry point; the swimming trajectory within $60 \mathrm{sec}$ was shot using a camera, the times across platform and the swimming time in original quadrant (target quadrant) were recorded.

Statistical analysis. Statistical analysis was performed using Statistical Product and Service Solutions (SPSS) 20.0 software (IBM Corp., Armonk, NY, USA). Measurement data are presented as mean \pm standard deviation; one-way analysis of variance (ANOVA) was used for the comparison among groups, and least-significant difference (LSD) t-test was used for the pairwise comparison. The repeated measures analysis of variance (ANOVA) was used for the data comparisons at different time points. $\mathrm{P}<0.05$ was considered to indicate a statistically significant difference.

\section{Results}

Expression levels of autophagy-associated proteins at different time points. The levels of LC3-I, LC3-II and Beclin-1 in hippocampal neurons and LC3-II/LC3-I value in sevoflurane group at $\mathrm{T} 2$ were significantly higher than those at $\mathrm{T} 1$, and the value was the highest at $\mathrm{T} 3$, but were decreased at T4 $(\mathrm{P}<0.05)$; the levels of proteins and LC3-II/LC3-I value in compound group at $\mathrm{T} 2$ were significantly higher than those at $\mathrm{T} 1$ and reached the peak, and remained unchanged at T3-T4; besides, the levels of proteins and LC3-II/LC3-I values in compound group at T2-T4 were significantly lower than those in sevoflurane group $(\mathrm{P}<0.05)$ (Figs. 1-3, Table I).

Escape latency, times across platform and swimming time in target quadrant of rats. In sevoflurane group, the escape latency was prolonged, the times across platform were 


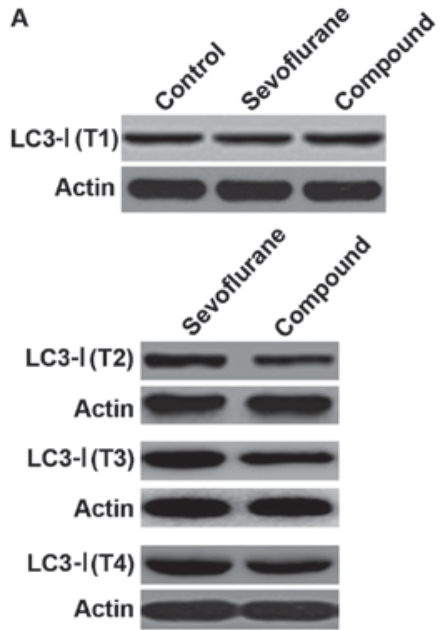

B

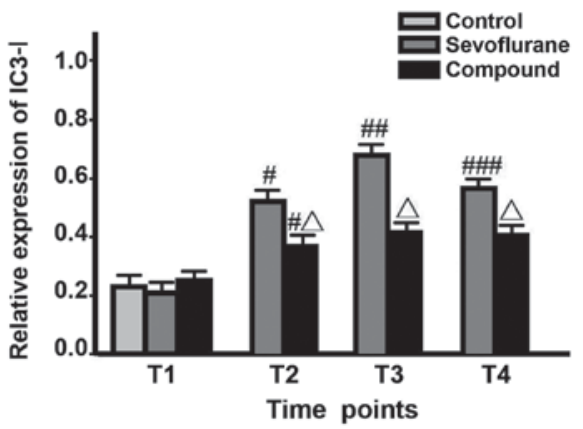

Figure 1. Detection of the LC3-I expression levels at different time points via western blotting $\left({ }^{\sharp} \mathrm{P}<0.05\right.$, comparisons of sevoflurane group and compound group at $\mathrm{T} 2$ with the same groups at $\mathrm{T} 1 ;{ }^{* \#} \mathrm{P}<0.05$, comparison between sevoflurane group at $\mathrm{T} 3$ and the same group at $\mathrm{T} 2$; ${ }^{\# \#} \mathrm{P}<0.05$, comparison between sevoflurane group at $\mathrm{T} 4$ and the same group at $\mathrm{T} 3 ;{ }^{\wedge} \mathrm{P}<0.05$, comparisons between compound group at $\mathrm{T} 2-\mathrm{T} 4$ and sevoflurane group in the same time)

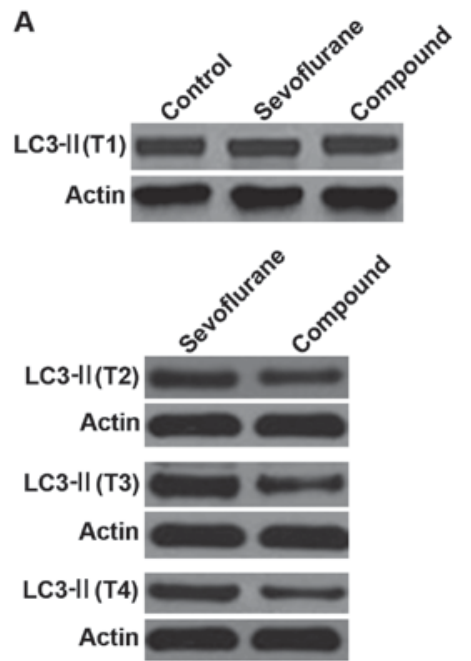

B

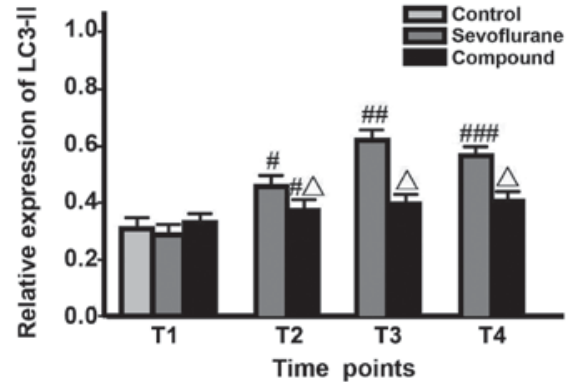

Figure 2. Detection of the LC3-II expression levels in different time via western blotting $\left({ }^{*} \mathrm{P}<0.05\right.$, comparisons of sevoflurane group and compound group at $\mathrm{T} 2$ with the same groups at $\mathrm{T} 1 ;{ }^{\# \#} \mathrm{P}<0.05$, comparison between sevoflurane group at $\mathrm{T} 3$ and the same group at $\mathrm{T} 2$; ${ }^{\# \#} \mathrm{P}<0.05$, comparison between sevoflurane group at $\mathrm{T} 4$ and the same group at $\mathrm{T} 3 ;{ }^{\wedge} \mathrm{P}<0.05$, comparisons between compound group at $\mathrm{T} 2-\mathrm{T} 4$ and sevoflurane group at the same time points).

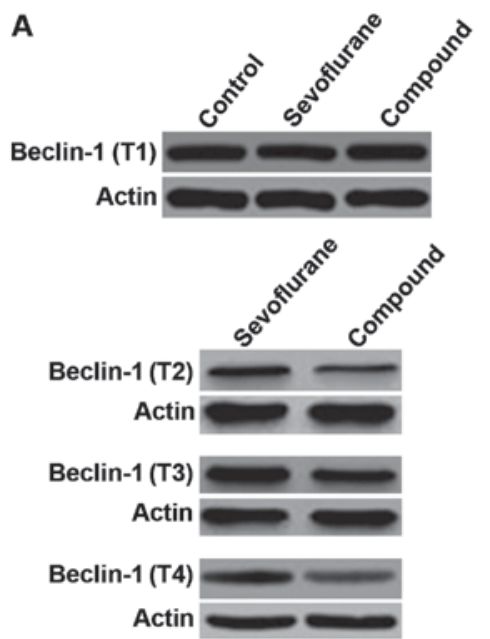

B

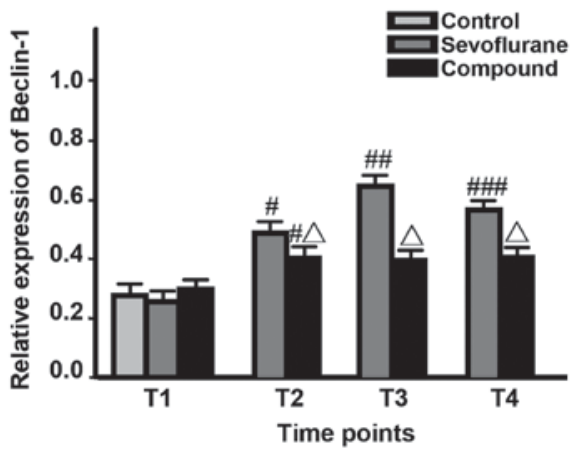

Figure 3. Detection of the Beclin-1 expression levels in different time via western blotting $\left({ }^{\#} \mathrm{P}<0.05\right.$, comparisons of sevoflurane group and compound group at $\mathrm{T} 2$ with the same groups at $\mathrm{T} 1 ;{ }^{\# \#} \mathrm{P}<0.05$, comparison between sevoflurane group at $\mathrm{T} 3$ and the same group at $\mathrm{T} 2$; ${ }^{\# \# \#} \mathrm{P}<0.05$, comparison between sevoflurane group at $\mathrm{T} 4$ and the same group at $\mathrm{T} 3 ;{ }^{\circ} \mathrm{P}<0.05$, comparisons between compound group at $\mathrm{T} 2-\mathrm{T} 4$ and sevoflurane group at the same time points). 
Table I. Comparison of LC3-II/LC3-I values at different time points.

\begin{tabular}{lcccc}
\hline Groups & $\mathrm{T} 1$ & $\mathrm{~T} 2$ & $\mathrm{~T} 3$ & $\mathrm{~T} 4$ \\
\hline Control & $1.05 \pm 0.26$ & - & - & - \\
Sevoflurane & $1.03 \pm 0.23$ & $0.89 \pm 0.13^{\mathrm{a}}$ & $0.92 \pm 0.15^{\mathrm{b}}$ & $0.77 \pm 0.14^{\mathrm{c}}$ \\
Compound & $1.07 \pm 0.28$ & $0.67 \pm 0.14^{\mathrm{a}, \mathrm{d}}$ & $0.68 \pm 0.16^{\mathrm{d}}$ & $0.66 \pm 0.12^{\mathrm{d}}$
\end{tabular}

${ }^{\mathrm{a}} \mathrm{P}<0.05$, comparison of sevoflurane group and compound group at $\mathrm{T} 2$ with the same groups at $\mathrm{T} 1$; ${ }^{\mathrm{b}} \mathrm{P}<0.05$, comparison between sevoflurane group at $\mathrm{T} 3$ and the same group at $\mathrm{T} 2{ }^{\mathrm{c}} \mathrm{P}<0.05$, comparison between sevoflurane group at $\mathrm{T} 4$ and the same group at $\mathrm{T} 3 ;{ }^{\mathrm{d}} \mathrm{P}<0.05$, comparisons between compound group at T2-T4 and sevoflurane group at the same time points.

Table II. Escape latency, times across platform and swimming time in target quadrant of rats.

\begin{tabular}{lccc}
\hline Groups & $\begin{array}{c}\text { Escape } \\
\text { latency (s) }\end{array}$ & $\begin{array}{c}\text { Times across } \\
\text { platform } \\
\text { (time) }\end{array}$ & $\begin{array}{c}\text { Swimming } \\
\text { time in target } \\
\text { quadrant (s) }\end{array}$ \\
\hline Control & $26.7 \pm 4.5$ & $2.3 \pm 0.3$ & $14.6 \pm 2.3$ \\
Sevoflurane & $33.5 \pm 4.9^{\mathrm{a}}$ & $1.6 \pm 0.3^{\mathrm{a}}$ & $10.4 \pm 2.1^{\mathrm{a}}$ \\
Compound & $28.2 \pm 4.6^{\mathrm{b}}$ & $2.1 \pm 0.4^{\mathrm{b}}$ & $13.2 \pm 2.4^{\mathrm{b}}$ \\
\hline
\end{tabular}

${ }^{\mathrm{a}} \mathrm{P}<0.05$, comparison between sevoflurane group and control group; ${ }^{\mathrm{b}} \mathrm{P}<0.05$, comparison between compound group and sevoflurane group.

reduced and the swimming time in target quadrant was shortened compared with those in control group $(\mathrm{P}<0.05)$; these parameters were significantly improved in compound group compared with those in sevoflurane group and control group $(\mathrm{P}<0.05)$ (Table II).

\section{Discussion}

The median effective dose of dexmedetomidine analgesic on rats is $1 \mu \mathrm{g} / \mathrm{kg}(8)$. Dexmedetomidine improves the occurrence of cognitive dysfunction after sevoflurane anesthesia, which may be related to the clearance of damaged mitochondria, delayed apoptosis, inhibition of cytochrome $c$ release, reduction of Caspase family activation, and reduction of apoptosis (9).

In this study, sevoflurane was used on anesthetized aged SD rats, and the effects of dexmedetomidine on hippocampal neurons autophagy and cognitive impairment in old rats were studied.

Morris water maze test is a common method to evaluate the spatial learning and memory ability of rodents (10). The results are reliable and repeatable. The study concluded that compared with rats in the control group, the rats in sevoflurane group had longer escape latency; they crossed the platform fewer times, and swam for a shorter period of time in the target quadrant. The condition of rats in the composite group was significantly better compared with that in sevoflurane group.
Therefore, we believe that dexmedetomidine can improve the cognitive dysfunction of sevoflurane anesthesia in aged rats.

Liu et al (11) showed that the sevoflurane anesthesiainduced POCD in aged rats is related to the $\beta$-amyloid deposit and Tau protein phosphorylation of hippocampal neurons. Satomoto et al (12) pointed out that the sevoflurane anesthesia can cause behavioral changes and cognitive abnormalities in young rats. Autophagy is a defense behavior of the body against the environmental stimulus, and the autophagosomes and specific proteins LC3-I, LC3-II and Beclin-1 can reflect the autophagic activity of the body (13). LC3-I can bind with the phosphatidylethanolamin on the surface of autophagy membrane after the ubiquitin-like modification, forming LC3-II (13), both of which are two protein products in the autophagy process, and the LC3-II/LC3-I value is better in reflecting the stability of autophagy. Beclin-1 is a specific gene involved in autophagy regulation in mammals.

The downregulation of Beclin-1 expression indicates the decline in autophagy, which is related to the anti-apoptotic protein Bcl-2 (14). Beclin-1 contains the BH3 structural domain, which binds Beclin-1 and Bcl-2 to inhibit Beclin-1-dependent autophagy-activated pathway through the interaction with Bcl-2 surface BH3. However, the combination of Beclin-1 and $\mathrm{Bcl}-2$ does not affect the fact that $\mathrm{Bcl}-2$ binds to the proapoptotic protein Bax, playing an anti-apoptotic effect, through the structural domain (15). The results of this study showed that the levels of LC3-I, LC3-II and Beclin-1 in hippocampal neurons and LC3-II/LC3-I value in sevoflurane group at T2 were significantly higher than those at $\mathrm{T} 1$, and they reached the peak at T3, but decreased at T4; the level of proteins and LC3-II/ LC3-I values in compound group at T2-T4 were obviously lower than those in sevoflurane group. Therefore, it is believed that sevoflurane anesthesia can up-regulate the autophagic activity of hippocampal neurons, and the anesthesia combined with dexmedetomidine can reduce the autophagic activity.

This study showed that the mechanism of sevoflurane anesthesia leading to the cognitive decline of rats was related to the increased autophagy of hippocampal neurons, providing an important reference basis for the clinical diagnosis and prevention of POCD. At the same time, the dexmedetomidine-assisted anesthesia can improve the cognitive function, providing an important idea for the application of dexmedetomidine.

\section{Acknowledgements}

Not applicable.

\section{Funding}

No funding was received.

\section{Availability of data and materials}

The datasets used and/or analyzed during the present study are available from the corresponding author on reasonable request.

\section{Authors' contributions}

CY conceived and designed the study, and drafted the manuscript. CY, ZF and XL collected, analyzed and interpreted the 
experimental data. ZF revised the manuscript for important intellectual content. All authors read and approved the final manuscript.

\section{Ethics approval and consent to participate}

The study was approved by the Animal Ethics Committee of Nanchang University (Nanchang, China).

\section{Consent for publication}

Not applicable.

\section{Competing interests}

The authors declare that they have no competing interests.

\section{References}

1. Tao G, Zhang J, Zhang L, Dong Y, Yu B, Crosby G, Culley DJ, Zhang Y and Xie Z: Sevoflurane induces tau phosphorylation and glycogen synthase kinase $3 \beta$ activation in young mice. Anesthesiology 121: 510-527, 2014.

2. Rundshagen I: Postoperative cognitive dysfunction. Dtsch Arztebl Int 111: 119-125, 2014.

3. Nixon RA: Autophagy, amyloidogenesis and Alzheimer disease. J Cell Sci 120: 4081-4091, 2007.

4. Galluzzi L, Pietrocola F, Bravo-San Pedro JM, Amaravadi RK, Baehrecke EH, Cecconi F, Codogno P, Debnath J, Gewirtz DA, Karantza V, et al: Autophagy in malignant transformation and cancer progression. EMBO J 34: 856-880, 2015.

5. Cai Y, Arikkath J, Yang L, Guo ML, Periyasamy P and Buch S: Interplay of endoplasmic reticulum stress and autophagy in neurodegenerative disorders. Autophagy 12: 225-244, 2016.

6. Whittington RA, Virág L, Gratuze M, Petry FR, Noël A, Poitras I, Truchetti G, Marcouiller F, Papon MA, El Khoury N, et al: Dexmedetomidine increases tau phosphorylation under normothermic conditions in vivo and in vitro. Neurobiol Aging 36: 2414-2428, 2015.
7. Ni J, Wei J, Yao Y, Jiang X, Luo L and Luo D: Effect of dexmedetomidine on preventing postoperative agitation in children: A meta-analysis. PLoS One 10: e0128450, 2015.

8. Sanders RD, Giombini M, Ma D, Ohashi Y, Hossain M, Fujinaga $M$ and Maze M: Dexmedetomidine exerts dosedependent age-independent antinociception but age-dependent hypnosis in Fischer rats. Anesth Analg 100: 1295-1302, 2005.

9. Zhu M, Wang H, Zhu A, Niu K and Wang G: Meta-analysis of dexmedetomidine on emergence agitation and recovery profiles in children after sevoflurane anesthesia: Different administration and different dosage. PLoS One 10: e0123728, 2015.

10. Zhang C, Hu J, Liu X and Yan J: Effects of intravenous dexmedetomidine on emergence agitation in children under sevoflurane anesthesia: A meta-analysis of randomized controlled trials PLoS One 9: e99718, 2014.

11. Liu W, Xu J, Wang H, Xu C, Ji C, Wang Y, Feng C, Zhang X, Xu Z, Wu A, et al: Isoflurane-induced spatial memory impairment by a mechanism independent of amyloid-beta levels and tau protein phosphorylation changes in aged rats. Neurol Res 34: 3-10, 2012.

12. Satomoto M, Satoh Y, Terui K, Miyao H, Takishima K, Ito M and Imaki J: Neonatal exposure to sevoflurane induces abnormal social behaviors and deficits in fear conditioning in mice. Anesthesiology 110: 628-637, 2009.

13. Zhou YF, Wang QX, Zhou HY and Chen G: Autophagy activation prevents sevoflurane-induced neurotoxicity in $\mathrm{H} 4$ human neuroglioma cells. Acta Pharmacol Sin 37: 580-588, 2016.

14. Liu K, Shi Y, Guo X, Wang S, Ouyang Y, Hao M, Liu D, Qiao L, Li N, Zheng J, et al: CHOP mediates ASPP2-induced autophagic apoptosis in hepatoma cells by releasing Beclin-1 from Bcl-2 and inducing nuclear translocation of Bcl-2. Cell Death Dis 5: e1323, 2014.

15. Trisciuoglio D, De Luca T, Desideri M, Passeri D, Gabellini C, Scarpino S, Liang C, Orlandi A and Del Bufalo D: Removal of the $\mathrm{BH} 4$ domain from $\mathrm{Bcl}-2$ protein triggers an autophagic process that impairs tumor growth. Neoplasia 15: 315-327, 2013.

This work is licensed under a Creative Commons Attribution-NonCommercial-NoDerivatives 4.0 International (CC BY-NC-ND 4.0) License. 